\title{
Sorting themselves out
}

How do the topographic maps that are characteristic of sensory brain regions form during development? Sakano and colleagues show that interactions between neuropilin 1 (NRP1) and its ligand semaphorin 3A (SEMA3A) regulate the organization of olfactory sensory neuron (OSN) axons long before they reach their targets in the olfactory bulb.

Cyclic AMP signalling is known to regulate the anterior-posterior positioning of OSN axon terminals in

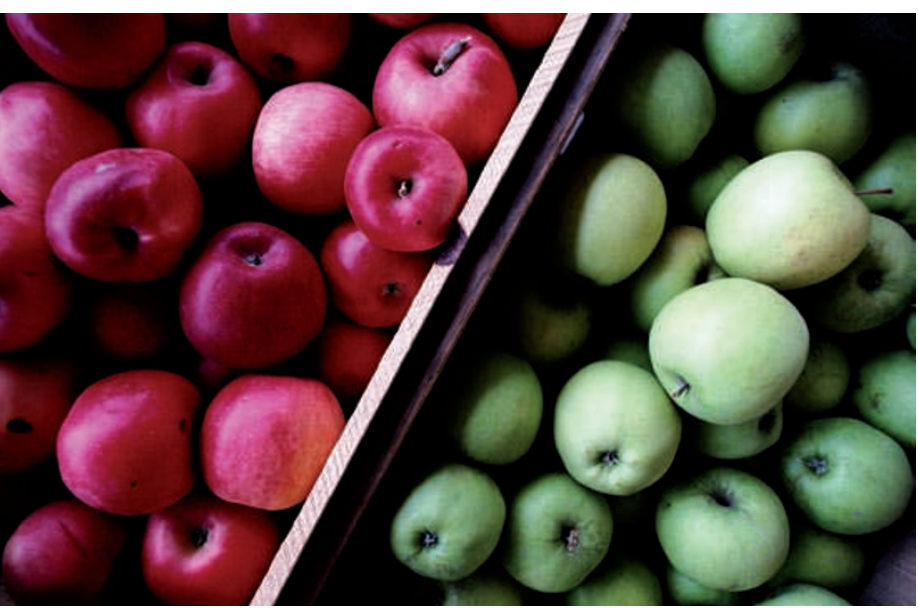

the olfactory bulb and cAMP levels in OSN axons correlate with NRP1 expression levels. The authors therefore investigated the role of NRP1 in mouse OSN organization.

The authors examined three axonal populations in the bundle of OSN axons projecting from the olfactory epithelium to the dorsal olfactory bulb: DI neurons, which express class I odorant receptors, and DII neurons, which express class II odorant receptors and are divided into DII-P (NRP1-positive) and DII-A (NRP1-negative) neurons. Although mixed as they initially project out of the olfactory epithelium, the authors found that the three populations of axons quickly sorted into three distinct domains within the bundle: medial (DI), central (DII-A) and lateral (DII-P).

When the authors knocked out NRP1 in a population of OSN neurons that are normally among the DII-P axons, the position of these axons was shifted centrally to the DII-A domain of the bundle. Conversely, when NRP1 was expressed in DII-A cells, their axons shifted laterally to the DII-P domain. These changes corresponded to anterior or posterior shifts in the position of the axon terminals in the olfactory bulb itself, suggesting that the axon's position in the bundle helps to shape map formation in the olfactory bulb.

The authors further showed that NRP1 and SEMA3A are expressed in a complementary pattern within the axonal bundle. Knocking out SEMA3A in OSNs disrupted the compartmentalization of DI, DII-A and DII-P axons in the bundle, indicating the importance of the NRP1-SEMA3A interaction for axonal sorting.

Thus, pre-target axonal sorting, mediated through NRP1-SEMA3A interactions, contributes to olfactory map formation. Similar axon-axon interactions might be a common feature of map formation in other sensory systems.

Katherine Whalley

ORIGINAL RESEARCH PAPER Imai, T. et al.

Pre-target axon sorting establishes the neural map topography. Science 9 Jul 2009 (doi:10.1126/ science.1173596) 